ARTICLE

https://doi.org/10.1038/s41467-019-11971-7

\title{
Giant oscillations in a triangular network of one- dimensional states in marginally twisted graphene
}

S.G. Xu (10) 1,2,5, A.I. Berdyugin (10) 1,5, P. Kumaravadivel (1) 1,2, F. Guinea1', R. Krishna Kumar 1,2, D.A. Bandurin',

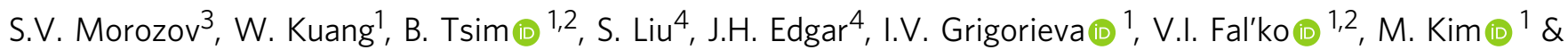
A.K. Geim (1) 1,2

At very small twist angles of $\sim 0.1^{\circ}$, bilayer graphene exhibits a strain-accompanied lattice reconstruction that results in submicron-size triangular domains with the standard, Bernal stacking. If the interlayer bias is applied to open an energy gap inside the domain regions making them insulating, such marginally twisted bilayer graphene is expected to remain conductive due to a triangular network of chiral one-dimensional states hosted by domain boundaries. Here we study electron transport through this helical network and report giant Aharonov-Bohm oscillations that reach in amplitude up to $50 \%$ of resistivity and persist to temperatures above $100 \mathrm{~K}$. At liquid helium temperatures, the network exhibits another kind of oscillations that appear as a function of carrier density and are accompanied by a signchanging Hall effect. The latter are attributed to consecutive population of the narrow minibands formed by the network of one-dimensional states inside the gap.

\footnotetext{
${ }^{1}$ School of Physics and Astronomy, University of Manchester, Manchester M13 9PL, UK. ${ }^{2}$ National Graphene Institute, University of Manchester, Manchester M13 9PL, UK. ${ }^{3}$ Institute of Microelectronics Technology and High Purity Materials, Russian Academy of Sciences, Chernogolovka 142432 , Russia. ${ }^{4}$ The Tim Taylor Department of Chemical Engineering, Kansas State University, Manhattan, KS 66506, USA. ${ }^{5}$ These authors contributed equally: S. G. Xu, A. I. Berdyugin. Correspondence and requests for materials should be addressed to M.K. (email: minsoo.kim@manchester.ac.uk) or to A.K.G. (email: geim@manchester.ac.uk)
} 
T he electronic properties of graphene superlattices have attracted intense interest ${ }^{1-4}$ that was further stimulated by the recent observation of novel many-body states in twisted bilayer graphene $(B L G)^{2,5-18}$. The latter system exhibits qualitative changes with varying the twist angle $\theta$ between the two graphene layers, which is caused by subtle interplay between the interlayer electron hybridization and the periodic crystallographic pattern known as a moiré superlattice $2,5-18$. For small $\theta$, the superlattice period is given by $\lambda=a /[2 \sin (\theta / 2)] \approx a / \theta$ and is much longer than graphene's lattice constant $a$. The recent interest in twisted BLG has been focused on so-called magic angles (typically, close to $\theta \approx 1.1^{\circ}$ ) at which the low-energy superlattice minibands become almost flat ${ }^{5,6}$ promoting electronelectron correlation effects and leading to unconventional insulating and superconducting states ${ }^{8-10}$. At the marginal twist angles, $\theta \ll 1^{\circ}$, the electronic structure is expected to become qualitatively different from that formed at magic or larger $\theta$ because the BLG superlattice undergoes a strain-accompanied lattice reconstruction such that there appear large (submicron) triangular domains with alternating Bernal ( $\mathrm{AB}$ and $\mathrm{BA}$ ) stacking order $^{11-13}$. The domain regions are rather similar to the conventional BLG and, if the displacement field $D$ is applied between the layers, a sizeable energy gap $\delta$ opens in the spectrum ${ }^{11,19,20}$, making the domains insulating ${ }^{19}$. Under these conditions, marginally twisted graphene (MTG) bilayers may still remain electrically conductive because walls between $\mathrm{AB}$ and $\mathrm{BA}$ domains allow one-dimensional (1D) chiral states ${ }^{11-18,21-24}$ (Fig. 1a). For an $\mathrm{AB} / \mathrm{BA}$ domain wall, there are four ( 2 spins and 2 valleys) gapless 1D states on each side. They propagate in opposite directions for different valleys and split apart at the superlattice's vertices where the stacking changes into AA (Fig. 1a). The unit block for this $2 \mathrm{D}$ network is an equilateral triangle with the area $A=\frac{\sqrt{3}}{4} \lambda^{2}$, half the size of the superlattice unit cell that includes both $\mathrm{AB}$ and $\mathrm{BA}$ domains.
In this Communication, we study the electronic properties of MTG and report exceptionally strong Aharonov-Bohm oscillations $\mathrm{s}^{25}$ arising from electron interference along the triangular loops forming the chiral network. Yet another type of oscillations is observed in MTG's resistivity as a function of carrier density, which indicates the presence of multiple electronic minibands inside the gap. Our work shows that marginally twisted BLG is markedly distinct from other 2D electronic systems, including BLG at larger twist angles, and offers a fascinating venue for further research.

\section{Results}

Experimental devices. Our devices were made from MTG that was prepared following the procedures developed in ref. ${ }^{26}$. In short, a monolayer graphene crystal was teared into two parts that were placed on top of each other by parallel transfer accompanied by small rotation. In our case the rotation angle $\theta$ was set close to zero (nominally, 0 to $0.1^{\circ}$ ). Subsequent transport measurements (see below) showed that the resulting bilayers exhibited twist angles of $\leq 0.25^{\circ}$. The MTG crystals were encapsulated in hexagonal boron nitride to improve their electronic quality and, using lithography techniques, shaped into dual-gated Hall bar devices such as shown Fig. 1b (Supplementary Note 1). The top gate was the standard metal-film electrode whereas the bottom gate was thin graphite, which further improved devices' electronic quality and reduced charge inhomogeneity. Four MTG devices were studied in detail, all exhibiting similar behavior. Below we focus on the results obtained for a device with $\theta \approx 0.10^{\circ}$ (Supplementary Note 2) which had the highest uniformity, as witnessed from practically the same magnetotransport characteristics observed using different contact configurations. For completeness, examples of the behavior observed for other MTG devices are provided in Supplementary Note 3.
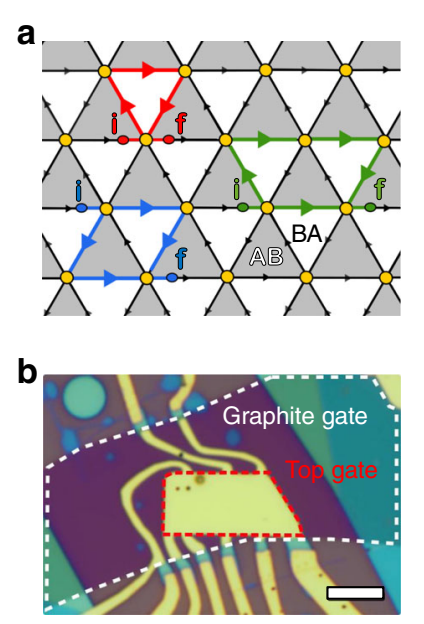

d
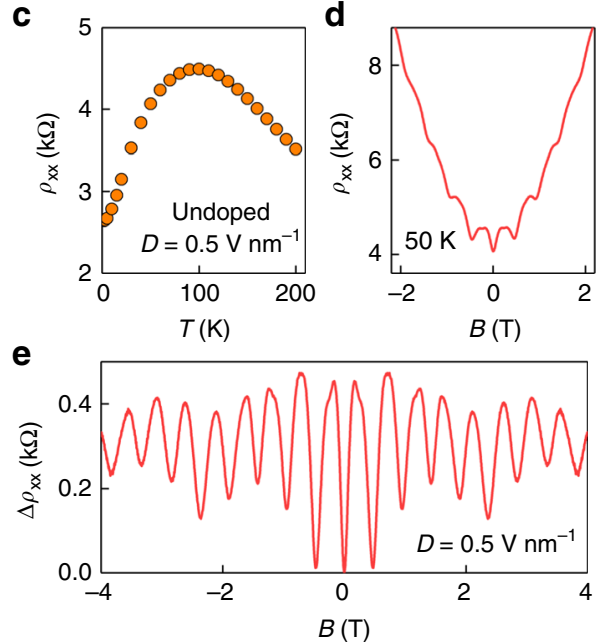

d

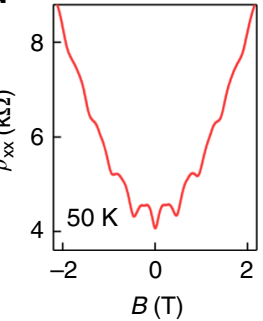

$B(\mathrm{~T})$

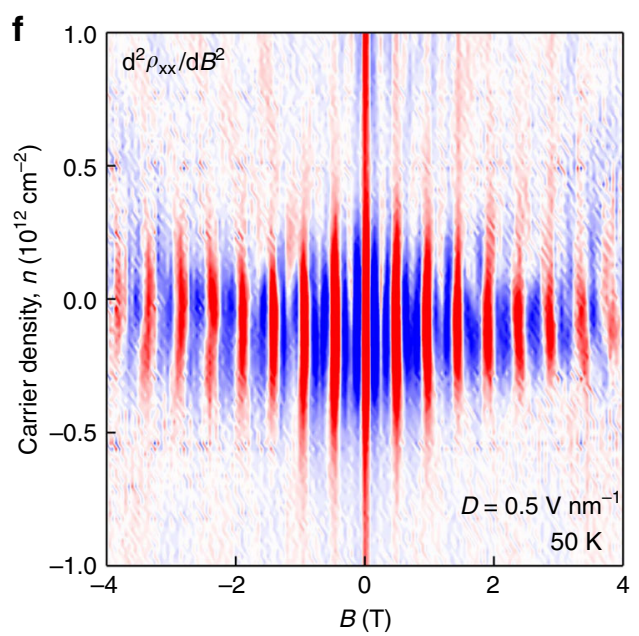

Fig. 1 Aharonov-Bohm oscillations in marginally twisted bilayer graphene. a Schematic of MTG: Its superlattice forms a triangular network of AB/BA domain walls ${ }^{11-13}$. The white and gray areas denote domains with the Bernal stacking; yellow circles-regions with AA stacking. Black arrows show the propagation direction for electrons in one of the valleys; colored arrows are examples of trajectories encircling one (red), two (blue), and three (green) triangular domains, which are responsible for different frequencies in Aharonov-Bohm oscillations. For example, the 2nd harmonic arises from interference between electrons propagating along the trajectories indicated by blue arrows (starting points are marked by ' $\mathbf{i}$ '; finishing by ' $\mathbf{f}$ '). b Optical micrograph of one of the studied devices. The graphite and top gates are indicated by color-coded dashed curves. The bright yellow regions are $\mathrm{Cr} / \mathrm{Au}$ contacts to graphene and the top gate. Scale bar, $5 \mu \mathrm{m}$. The device exhibited carrier mobility of $\sim 10^{4} \mathrm{~cm}^{2} \mathrm{~V}^{-1} \mathrm{~s}^{-1}$ at $n \approx 10^{12} \mathrm{~cm}^{-2}$, which is typical for small twist angles ${ }^{12,16}$ where irregularity in positions of domain walls probably causes additional scattering. $\mathbf{c}$ Temperature dependence of undoped MTG with the energy gap of $\sim 50 \mathrm{meV}$ induced by interlayer bias. $\mathbf{d}$ Magnetoresistance at the NP for the same device at $50 \mathrm{~K}$. e Same as $\mathbf{d}$ but a monotonic background is subtracted for clarity. $\mathbf{f}$ Same Aharonov-Bohm oscillations for different doping. Instead of subtracting the magnetoresistance for each $n$ as in $\mathbf{e}$, we plot $\mathrm{d}^{2} \rho_{\mathrm{xx}}(B) / \mathrm{d} B^{2}$ which removes the smooth background without shifting positions of oscillations' extrema. Blue-to-red scale, $\pm 4 \mathrm{kOhm} \mathrm{T}^{-2}$ 
1D conductive network. To study electron transport through the expected network created by $\mathrm{AB} / \mathrm{BA}$ domain walls, we applied the displacement field $D$ using the top and bottom gates (Supplementary Note 1), which opened an energy gap in the Bernalstacked regions ${ }^{11,19,20}$. For $D=0.5 \mathrm{~V} \mathrm{~nm}^{-1}$ (achievable without a risk of damaging the devices), the gap $\delta$ inside $\mathrm{AB}$ and $\mathrm{BA}$ regions should be ${ }^{20} \sim 50 \mathrm{meV}$ so that they become highly insulating at temperatures $T \leq 50 \mathrm{~K}$ as known from the experiments on standard $\mathrm{BLG}^{19}$. In contrast, our MTG devices exhibited a distinctly metallic behavior such that longitudinal resistivity $\rho_{\mathrm{xx}}$ decreased with decreasing $T$, reaching a few $\mathrm{kOhms}$ at liquid-helium $T$ (Fig. 1c). This shows that, at the charge neutrality point (NP), MTG hosts a metallic system (Supplementary Note 5), in contrast to Bernal-stacked BLG and in agreement with the expected 1D transport along $\mathrm{AB} / \mathrm{BA}$ walls. At $T>100 \mathrm{~K}$, the temperature behavior changed so that $\rho_{\mathrm{xx}}$ decreased with increasing $T$ (Fig. 1c). The latter observation is attributed to thermally activated carriers in the gapped $\mathrm{AB}$ and $\mathrm{BA}$ regions, similar to the case of standard BLG ${ }^{19}$. The insulating behavior could also be suppressed by field-effect doping. For $D=0.5 \mathrm{~V} \mathrm{~nm}^{-1}$, it typically required carrier densities $n$ above $\pm 3-5 \times 10^{11} \mathrm{~cm}^{-2}$ to start populating the conduction and valence bands, as seen from a drop in $\rho_{\mathrm{xx}}$ (Supplementary Note 4 ).

Aharonov-Bohm oscillations. The network of conducting $\mathrm{AB} /$ BA walls revealed itself most clearly in strong magnetooscillations that were periodic in magnetic field $B$ and observed for all our MTG devices. Examples are shown in Fig. 1d-f and Supplementary Fig. 2c. The oscillations developed with increasing $D$ and persisted until $\mathrm{AB}$ and $\mathrm{BA}$ domains became conductive because of either doping or temperature. For example, at $D=0.5 \mathrm{~V} \mathrm{~nm}^{-1}$, this meant $|n|$ up to $5 \times 10^{11} \mathrm{~cm}^{-2}$ and $T$ up to $120 \mathrm{~K}$ as seen in Fig. If and Fig. 2, respectively. The periodic-in- $B$ oscillations persisted up to several Tesla where they became overwhelmed by Shubnikov-de Haas oscillations (Supplementary Note 3 ). We attribute the low- $B$ oscillations to the Aharonov-Bohm effect ${ }^{25}$ for electrons propagating along the triangular network of $1 \mathrm{D}$ channels hosted by $\mathrm{AB} / \mathrm{BA}$ walls. Indeed, interference between electronic states propagating along, e.g., the red loop in Fig. 1a is expected to vary periodically with the magnetic flux piercing the domain area $A$. For the particular

a

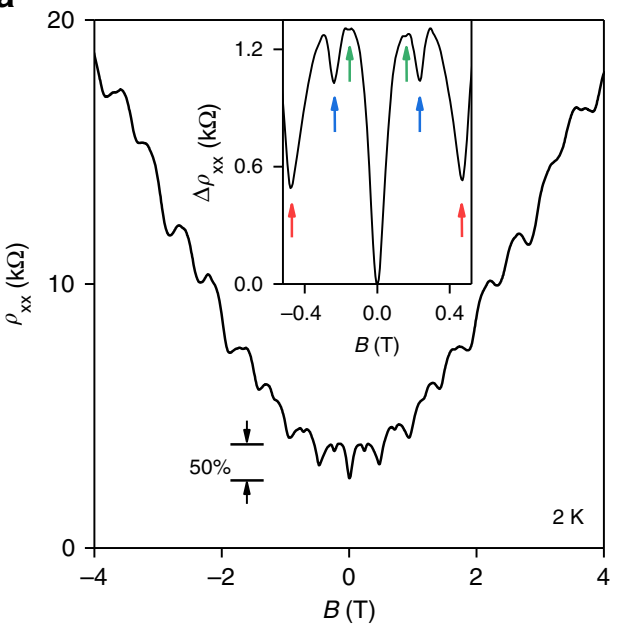

device in Fig. 1, we found the oscillation period $\Delta B \approx 0.48 \pm 0.02$ $\mathrm{T}$ which translates into one flux quantum $\phi_{0}=h / e$ per $A \approx$ $0.86 \pm 0.04 \times 10^{-10} \mathrm{~cm}^{2}$ and yields $\lambda \approx 140 \mathrm{~nm}$ or $\theta \approx 0.10^{\circ} \pm$ $3 \%$, in agreement with the area found from the position of superlattice NPs (Supplementary Note 2).

As $T$ decreased below $50 \mathrm{~K}$, the Aharonov-Bohm oscillations grew exponentially, reaching more than $1 \mathrm{kOhm}$ in amplitude at liquid-helium $T$, that is, nearly $\sim 50 \%$ of zero- $B$ resistivity (Fig. 2a). Furthermore, higher frequency harmonics became visible at low $T$ (inset of Fig. 2a). For quantitative analysis, $\rho_{\mathrm{xx}}(B)$ curves for a given $T$ were Fourier-transformed (see the inset of Fig. $2 \mathrm{~b}$ ). The peaks in the Fourier plot reveal the main periodicity $\Delta B \approx 0.48 \mathrm{~T}$ (same as in Fig. 1) plus two fractional periods, $\Delta B / 2$ and $\Delta B / 3$. The latter correspond to twice and thrice larger areas involved in the interference pattern and can be attributed to the loops such as those indicated by the blue and green arrows in Fig. 1a. This assignment agrees well with the fact that the higher harmonics decayed notably faster with increasing $T$ than the main-frequency oscillations (Fig. 2b), as expected because of the larger circumferences of the blue and green loops. Moreover, the suppression of Aharonov-Bohm oscillations is usually described by the dependence ${ }^{27} \exp \left(-L / L_{\phi}\right)$ where $L$ is the length of the interference loops, and $L_{\phi}(T)$ is the decoherence length. The color-coded lines in Fig. $2 \mathrm{~b}$ show that the decay rates for the 1st, 2nd, and 3rd frequencies followed the ratios $L / L_{\phi}$ expected from the circumferences of the three involved loops $(L=3 \lambda, 4 \lambda$, and $5 \lambda$, respectively) for a given $L_{\phi}(T)$.

Minibands inside the gap. We also studied the network's transport properties as a function of doping, keeping $n$ sufficiently low to remain in the insulating state for the $\mathrm{AB}$ and $\mathrm{BA}$ domains. Figure 3 shows that our MTG devices exhibited strong oscillations in their zero- $B$ resistivity as a function of $n$. This oscillatory behavior was not due to mesoscopic (interference) fluctuations ${ }^{28}$ : It remained the same for measurements using different contact configurations and different $D$. The oscillations were even more profound in Hall resistivity $\rho_{\mathrm{xy}}(n)$ that reversed its sign many times within the gapped region (Fig. $3 \mathrm{~b}$ and Supplementary Fig. 2b). The observed behavior can be understood as changes in the global interference pattern with varying $n$ and, hence, the Fermi wavelength. This is conceptually similar

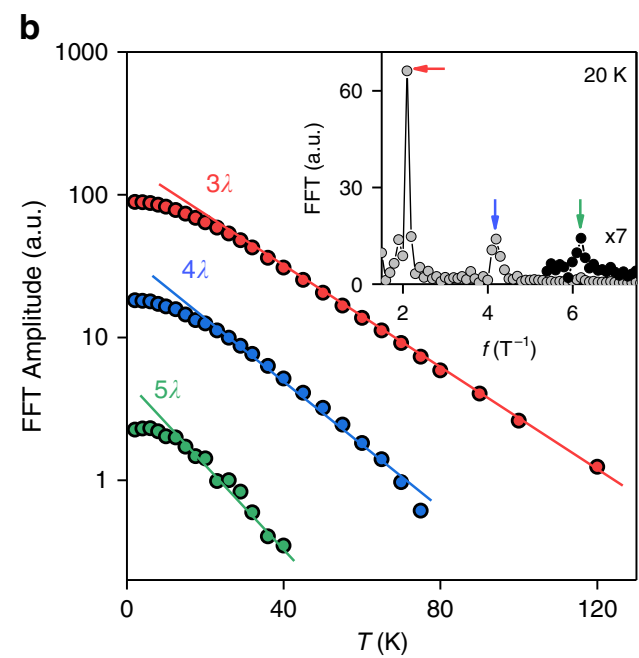

Fig. 2 High-order harmonics in the Aharonov-Bohm oscillations. a Typical magnetoresistance curves near the main $N P\left(T=2 K_{,} D=0.5 \vee n m^{-1}\right)$. Insert: Zoom-in close to zero $B$. The arrows mark the minima that correspond to $\phi_{0}$ piercing one (red), two (blue) and three (green) triangular domains shown in Fig. 1a. b Temperature dependence for the three oscillation frequencies (symbols). Solid lines: Guides to the eye indicate that the slopes for the 2 nd and 3rd harmonics are, respectively, 4/3 and 5/3 times steeper than for the main frequency. Insert: Example of our fast-Fourier-transform (FFT) analysis (gray circles). The peaks are marked using the same color coding as in $\mathbf{a}$. The third-harmonic peak is magnified for clarity (black symbols) 
a

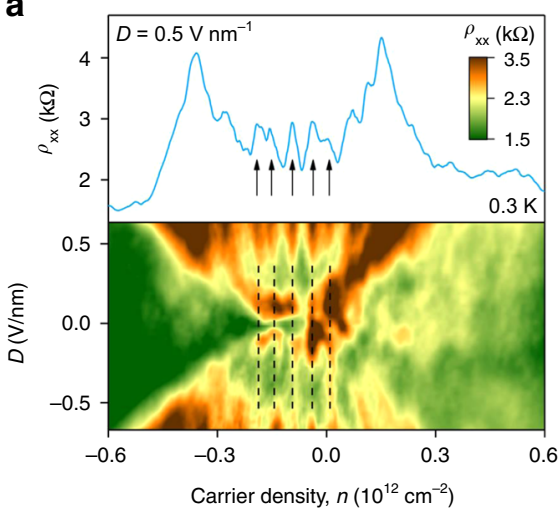

b

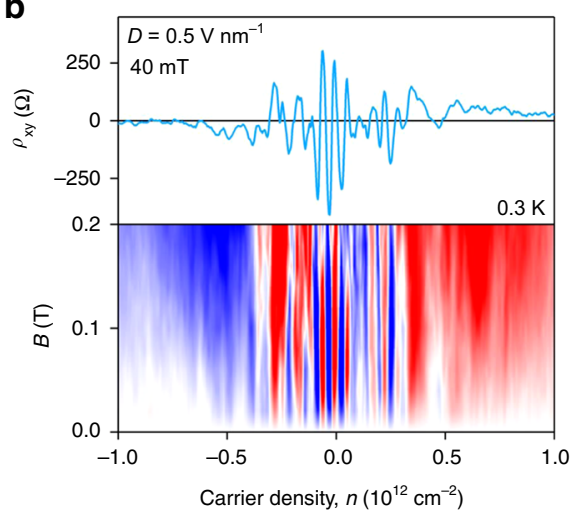

C
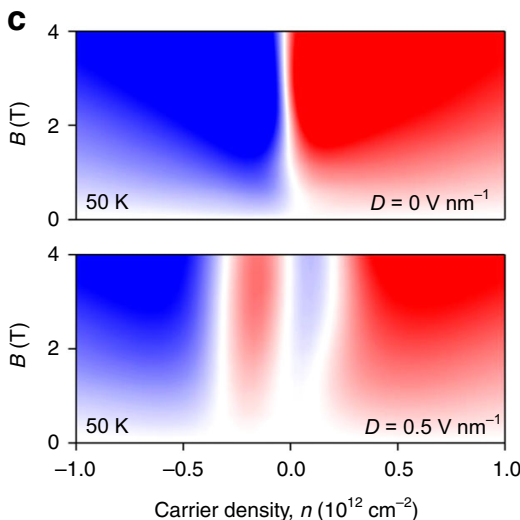

Fig. 3 Minibands in the gapped state of marginally twisted BLG. a Longitudinal resistivity as a function of $n$ and $D$ at 0.3 K. Upper panel: Crosscut of the map at $D=+0.5 \mathrm{~V} \mathrm{~nm}^{-1}$. b Same oscillatory behavior in Hall resistivity as a function of $n$ and $B$ for the given $D$. Color scale: \pm 250 Ohm. Upper panel: Crosscut at

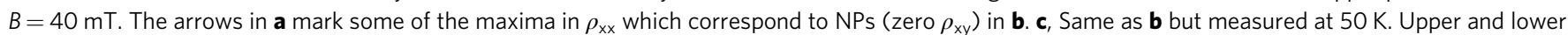
panels are for $D=0$ and $0.5 \mathrm{~V} \mathrm{~nm}^{-1}$, respectively. Color scale: $\pm 3 \mathrm{kOhm}$

to Aharonov-Bohm oscillations that are caused by the periodicin- $B$ phase modulation. The expected main periodicity $\Delta n$ is given $7,8,16$ by $4 / A$ which corresponds to one extra electron per $\mathrm{AB}$ or $\mathrm{BA}$ domain, taking into account the fourfold degeneracy. Figure $3 \mathrm{a}, \mathrm{b}$ yield characteristic $\Delta n \approx 5 \pm 0.5 \times 10^{10} \mathrm{~cm}^{-2}$ and, hence, $A \approx 0.80 \pm 0.09 \times 10^{-10} \mathrm{~cm}^{2}$, which agrees well with the area $0.86 \pm 0.04 \times 10^{-10} \mathrm{~cm}^{2}$ found from the main period of Aharonov-Bohm oscillations. The observed oscillations in $n$ can equally be interpreted as the consecutive filling of electronic minibands formed by the triangular $2 \mathrm{D}$ lattice of $1 \mathrm{D}$ chiral states as suggested in refs. ${ }^{14,15}$ (also, see Supplementary Note 5). The electronic spectrum for each such miniband has both electron and hole like states, which should lead to multiple NPs and signchanging $\rho_{\mathrm{xy}}(n)$. Accompanying oscillations in $\rho_{\mathrm{xx}}(n)$ are also expected to appear as the minibands are filled one by one. The number of the observed minibands can be estimated by counting the number of NPs (for example, in Fig. 3b there are about 10 oscillations inside the gapped region $\left(|n|<3 \times 10^{11} \mathrm{~cm}^{-2}\right)$. As $D=0.5 \mathrm{~V} \mathrm{~nm}^{-1}$ results in $\delta \approx 50 \mathrm{meV}^{20}$, the average width $\varepsilon$ of those minibands is $\sim 5 \mathrm{meV}$.

\section{Discussion}

The reason why the minibands evolve periodically in $B$ (Aharonov-Bohm oscillations) but not fully so in $n$ could be the following. First, the interlayer bias $D$ and finite $n$ remove the degeneracy between $\mathrm{AB}$ and $\mathrm{BA}$ domains so that $1 \mathrm{D}$ states propagating at the opposite sides of $\mathrm{AB} / \mathrm{BA}$ walls acquire somewhat different Fermi velocities ${ }^{29}$. This should lead to beatings between interference oscillations arising from $\mathrm{AB}$ and $\mathrm{BA}$ domains. Interference along the longer loops should further contribute to some randomness in the oscillatory behavior. To elucidate the mentioned analogy between oscillations caused by changing the Fermi wavelength and the miniband description, we estimate the energy difference $\Delta E=h v_{\mathrm{DW}} / L$ between the states arising from consecutive standing waves in the smallest loop $L=3 \lambda$ where $h$ is the Planck constant and $v_{\mathrm{DW}}$ is the drift velocity of the $1 \mathrm{D}$ states at $\mathrm{AB} / \mathrm{BA}$ walls. After taking into account the lifted degeneracy between $\mathrm{AB}$ and $\mathrm{BA}$ domains, the experimental value for $\Delta E$ is $2 \varepsilon \approx 10 \mathrm{meV}$, which yields $v_{\mathrm{DW}} \approx 10^{6} \mathrm{~m} \mathrm{~s}^{-1}$, comparable to graphene's Dirac velocity. Such a large value of $v_{\mathrm{DW}}$ suggests extremely sharp boundaries between AB and BA domains (Supplementary Note 6), which agrees with the strain reconstruction for small- $\theta$ superlattices as found by electron microscopy ${ }^{12}$ and infrared nano-imaging ${ }^{13}$.
Finally, let us point out some other surprising features of electron transport through the $\mathrm{AB} / \mathrm{BA}$ domain network. First, the oscillations caused by filling the minibands are rapidly smeared by $T$ and completely disappear above $20 \mathrm{~K}$ in both $\rho_{\mathrm{xx}}$ and $\rho_{\mathrm{xy}}$ (Supplementary Note 4 and Fig. $3 \mathrm{c}$ ). This is probably expected as the minibands are only $5 \mathrm{meV}$ apart and, therefore, cannot be resolved at such high $T$. In contrast, the Aharonov-Bohm oscillations survive to much higher $T$ (Fig. 2). On one hand, this is perhaps not surprising because of relatively long $L_{\phi}$ typical for graphene. On the other hand, the robustness seemingly contradicts to the narrow minibands description. To explain this conundrum, we refer to Brown-Zak oscillations ${ }^{30}$ that also have their origins in the Aharonov-Bohm effect but appear for superlattices with $2 \mathrm{D}$ conductivity rather than in a network of conductive 1D states. Brown-Zak oscillations in graphene superlattices were found to be exceptionally robust and survived above room $T$, despite the thermal smearing covered many minibands ${ }^{30}$. This is because the minibands respond to $B$ in a uniform manner, which was described in terms of changing the average group velocity ${ }^{30}$. A similar description is likely to be applicable to the narrow minibands in MTG and explain the robust Aharonov-Bohm oscillations for the thermally smeared minibands.

Another puzzle is the reversal of the average Hall effect found for our conductive network. This is shown in Fig. $3 \mathrm{c}$ where $\rho_{\mathrm{xy}}$ is plotted for $D=0$ and $0.5 \mathrm{~V} \mathrm{~nm}^{-1}$ at $50 \mathrm{~K}$. Without an interlayer bias (top panel), the Hall response is normal, with positive $\rho_{\mathrm{xy}}$ for electrons and negative for holes (for a given $B$ direction). In contrast, as the triangular network was formed by applying the interlayer bias, the Hall effect reversed its sign. The normal behavior recovered only at high $n$, inside the conduction and valence bands. The reversal of the average sign of $\rho_{\mathrm{xy}}$ implies that the network's minibands are predominantly hole-like for electron doping and vice versa for hole doping. This observation does not follow from any of the existing models ${ }^{14,15}$. To understand the reversal qualitatively, we evoke an analogy with $1 \mathrm{D}$ chiral states in the quantum Hall effect. From a semiclassical perspective, these states can be viewed as skipping orbits and, along the inner boundaries of $\mathrm{AB}$ and $\mathrm{BA}$ domains, electrons would then circulate in the direction opposite to that for cyclotron orbits, that is, an average effect from such small closed loops would be hole-like. Accordingly, a collection of skipping orbits on a triangular network may result in a hole-like Hall effect. This analogy requires theoretical corroboration in terms of minibands' electronic spectra of MTG. 


\section{Methods}

Device fabrication. Our MTG samples were encapsulated between two hBN crystals and prepared using the tear-and-stack method (see Supplementary Note 1 for more details). We used few-layer graphite as the back gate to reduce charge inhomogeneity. The final devices were shaped into the Hall bar geometry using electron-beam lithography and reactive-ion etching.

Electrical measurements. The measurements were carried out using the standard low-frequency lock-in technique with excitation frequencies of 6 to $30 \mathrm{~Hz}$ and currents of typically $10 \mathrm{nA}$ at $0.3 \mathrm{~K}$ and $100 \mathrm{nA}$ at higher temperatures.

\section{Data availability}

The data that support the findings of this study are available from the corresponding author upon reasonable request.

Received: 4 April 2019 Accepted: 12 August 2019

Published online: 05 September 2019

\section{References}

1. Yankowitz, M., Ma, Q., Jarillo-Herrero, P. \& LeRoy, B. J. van der Waals heterostructures combining graphene and hexagonal boron nitride. Nat. Rev. Phys. 1, 112-125 (2019).

2. Li, G. et al. Observation of Van Hove singularities in twisted graphene layers. Nat. Phys. 6, 109-113 (2010)

3. Ponomarenko, L. A. et al. Cloning of Dirac fermions in graphene superlattices. Nature 497, 594-597 (2013).

4. Dean, C. R. et al. Hofstadter's butterfly and the fractal quantum Hall effect in moiré superlattices. Nature 497, 598-602 (2013).

5. Suárez Morell, E., Correa, J. D., Vargas, P., Pacheco, M. \& Barticevic, Z. Flat bands in slightly twisted bilayer graphene: Tight-binding calculations. Phys. Rev. B 82, 121407(R) (2010).

6. Bistritzer, R. \& MacDonald, A. H. Moiré bands in twisted double-layer graphene. Proc. Natl Acad. Sci. 108, 12233-12237 (2011).

7. Kim, K. et al. Tunable moiré bands and strong correlations in small-twistangle bilayer graphene. Proc. Natl Acad. Sci. 114, 3364-3369 (2017).

8. Cao, Y. et al. Correlated insulator behaviour at half-filling in magic-angle graphene superlattices. Nature 556, 80-84 (2018).

9. Cao, Y. et al. Unconventional superconductivity in magic-angle graphene superlattices. Nature 556, 43-50 (2018).

10. Yankowitz, M. et al. Tuning superconductivity in twisted bilayer graphene. Science 363, 1059-1064 (2019).

11. Huang, S. et al. Topologically protected helical states in minimally twisted bilayer graphene. Phys. Rev. Lett. 121, 037702 (2018).

12. Yoo, H. et al. Atomic and electronic reconstruction at the van der Waals interface in twisted bilayer graphene. Nat. Mater. 18, 448-453 (2019).

13. Sunku, S. S. et al. Photonic crystals for nano-light in moiré graphene superlattices. Science 362, 1153-1156 (2018).

14. San-Jose, P. \& Prada, E. Helical networks in twisted bilayer graphene under interlayer bias. Phys. Rev. B 88, 121408(R) (2013).

15. Efimkin, D. K. \& Macdonald, A. H. Helical network model for twisted bilayer graphene. Phys. Rev. B 98, 035404 (2018).

16. Rickhaus, P. et al. Transport through a network of topological channels in twisted bilayer graphene. Nano Lett. 18, 6725-6730 (2018).

17. Ramires, A. \& Lado, J. L. Electrically tunable gauge fields in tiny-angle twisted bilayer graphene. Phys. Rev. Lett. 121, 146801 (2018).

18. Wu, X. C., Jian, C. M. \& Xu, C. Coupled-wire description of the correlated physics in twisted bilayer graphene. Phys. Rev. B 99, 161405(R) (2019).

19. Oostinga, J. B., Heersche, H. B., Liu, X., Morpurgo, A. F. \& Vandersypen, L. M. K. Gate-induced insulating state in bilayer graphene devices. Nat. Mater. 7, 151-157 (2008).

20. Zhang, Y. et al. Direct observation of a widely tunable bandgap in bilayer graphene. Nature 459, 820-823 (2009).

21. Martin, I., Blanter, Y. M. \& Morpurgo, A. F. Topological confinement in bilayer graphene. Phys. Rev. Lett. 100, 036804 (2008).

22. Ju, L. et al. Topological valley transport at bilayer graphene domain walls. Nature 520, 650-655 (2015)
23. Yin, L. J., Jiang, H., Qiao, J. Bin \& He, L. Direct imaging of topological edge states at a bilayer graphene domain wall. Nat. Commun. 7, 11760 (2016).

24. Li, J. et al. Gate-controlled topological conducting channels in bilayer graphene. Nat. Nanotechnol. 11, 1060-1065 (2016).

25. Webb, R. A., Washburn, S., Umbach, C. P. \& Laibowitz, R. B. Observation of h/e Aharonov-Bohm Oscillations in Normal-Metal Rings. Phys. Rev. Lett. 54, 2696-2699 (1985)

26. Kim, K. et al. van der Waals heterostructures with high accuracy rotational alignment. Nano Lett. 16, 1989-1995 (2016).

27. Hansen, A. E., Kristensen, A., Pedersen, S., Sørensen, C. B. \& Lindelof, P. E. Mesoscopic decoherence in Aharonov-Bohm rings. Phys. Rev. B 64, 045327 (2001).

28. Beenakker, C. W. J., Houten, H. \& van Quantum transport in semiconductor nanostructures. Solid State Phys. 44, 1-228 (1991).

29. Lane, T. L. M. et al. Ballistic electron channels including weakly protected topological states in delaminated bilayer graphene. Phys. Rev. B 97, 045301 (2018).

30. Krishna Kumar, R. et al. High-order fractal states in graphene superlattices. Proc. Natl Acad. Sci. 115, 5135-5139 (2018).

\section{Acknowledgements}

This work was supported by Graphene Flagship, the Royal Society and Lloyd's Register Foundation. S.V.M. was supported by Russian Science Foundation (grant 17-12-01393), and R.K.K. by an EPSRC fellowship award. S.L. and J.H.E. acknowledge support from the Materials Engineering and Processing program of the National Science Foundation (award CMMI 1538127) and the II-VI Foundation. M.K. was supported by National Research Foundation of Korea (Grant 2018R1A6A3A03010943). A.I.B., W.K., B.T. were supported by Graphene NowNANO Doctoral Training Programme.

\section{Author contributions}

S.G.X. and P.K. fabricated the devices. A.I.B. and M.K. carried out electrical measurements with help from R.K.K., S.V.M., D.A.B., and W.K. The results were analyzed by A.I.B. and M.K. with help from S.G.X., R.K.K., B.T., V.I.F., and A.K.G. V.I.F. and F.G. provided the presented theory calculations. Hexagonal boron nitride crystals were grown by S.L. and J.H.E. A.K.G. and A.I.B. wrote the paper with contributions from S.G.X., M.K., P.K., I.V.G., and V.I.F. All authors contributed to the discussions.

\section{Additional information}

Supplementary Information accompanies this paper at https://doi.org/10.1038/s41467 019-11971-7.

Competing interests: The authors declare no competing interests.

Reprints and permission information is available online at http://npg.nature.com/ reprintsandpermissions/

Peer review information: Nature Communications thanks Eslam Khalaf and other anonymous reviewer(s) for their contribution to the peer review of this work.

Publisher's note: Springer Nature remains neutral with regard to jurisdictional claims in published maps and institutional affiliations.

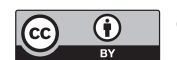

Open Access This article is licensed under a Creative Commons Attribution 4.0 International License, which permits use, sharing, adaptation, distribution and reproduction in any medium or format, as long as you give appropriate credit to the original author(s) and the source, provide a link to the Creative Commons license, and indicate if changes were made. The images or other third party material in this article are included in the article's Creative Commons license, unless indicated otherwise in a credit line to the material. If material is not included in the article's Creative Commons license and your intended use is not permitted by statutory regulation or exceeds the permitted use, you will need to obtain permission directly from the copyright holder. To view a copy of this license, visit http://creativecommons.org/ licenses/by/4.0/.

(c) The Author(s) 2019 